\title{
THE GLAUCOMAS
}

A R Elkington, P T Khaw

\section{Symptoms and signs}

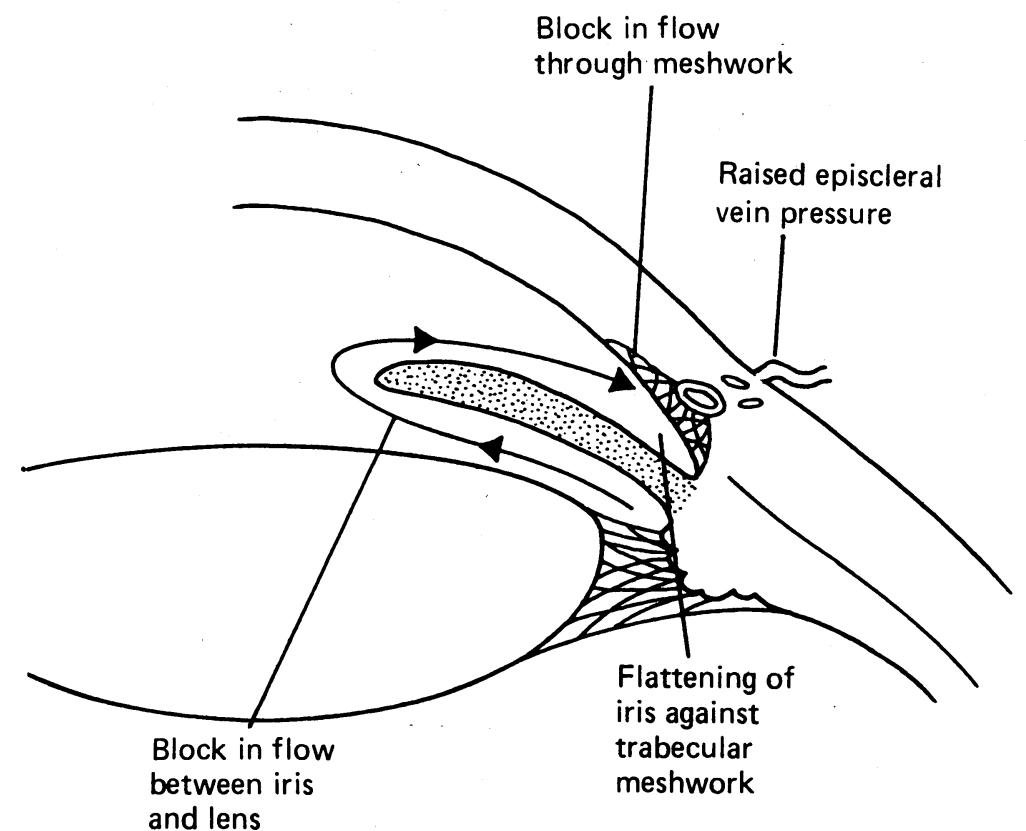

The glaucomas are a range of disorders that are characterised by optic disc cupping, visual field loss, and an intraocular pressure sufficiently raised to damage the eye.

Normally the ciliary body secretes aqueous, which then flows through the posterior chamber and through the pupil into the anterior chamber. It then leaves the eye through the trabecular meshwork, flowing into the canal of Schlemm. The flow and drainage can be obstructed in several ways.

Normal aqueous drainage and possible sites of obstruction.

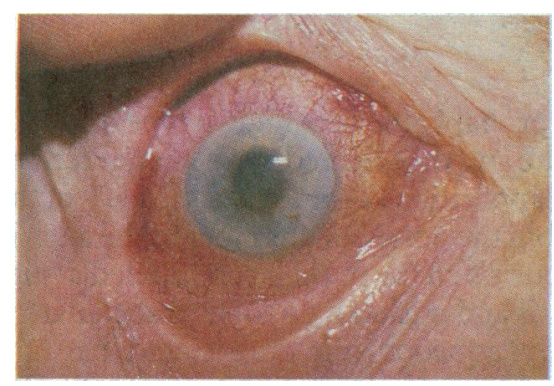

Cloudy cornea after sudden rise in intraocular pressure (acute angle closure glaucoma).

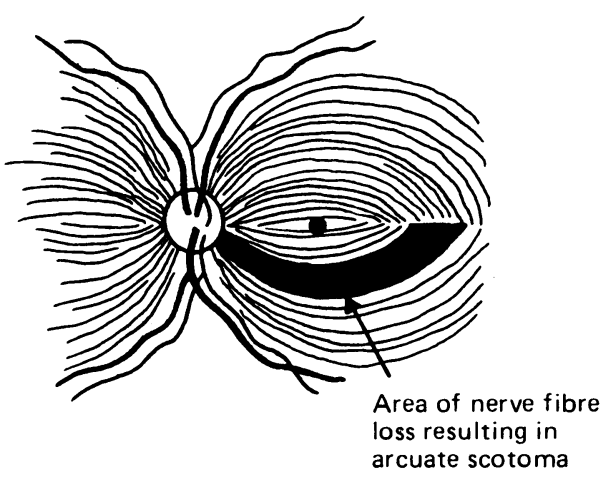

Normal distribution of nerve fibres in the retina.
The patient with chronic open angle glaucoma may not notice any symptoms until severe visual damage has occurred. This is because the rise in pressure and consequent damage occur so slowly that the patient has time to compensate. In contrast, the clinical presentation of acute angle closure glaucoma is well known as the intraocular pressure rises rapidly and results in a red, painful eye with disturbance of vision.

Haloes around lights and a cloudy cornea - The cornea is kept transparent by the continuous removal of fluid by the endothelial cells. If the pressure rises slowly this process takes longer to fail. When the pressure rises quickly (acute closed angle glaucoma) the cornea becomes waterlogged, causing a fall in visual acuity and giving rise to the symptom of haloes (analogous to looking through frosted glass).

Pain-If the rise in pressure is slow pain is not a feature of glaucoma until the pressure is extremely high. Pain is not a feature of chronic open angle glaucoma.

Field loss-The normal distribution of the retinal nerve fibres is shown. Pressure on the nerve fibres and chronic ischaemia at the head of the nerve cause damage to these fibres and usually result in a characteristic pattern of field loss (arcuate scotoma). This, however, spares central vision initially, and the patient may not notice the defect. The terminal stage of glaucomatous field loss is a field that is severely contracted with only a few remaining fibres from the more richly innervated macular area surviving. Even at this stage the vision may still be $6 / 6$ with virtually no visual field remaining.

Disc changes - The optic disc marks the exit point of the retinal nerve fibres from the eye. In the presence of a sustained rise in pressure the nerve fibres atrophy, leaving the characteristic cupped disc of chronic glaucoma. 


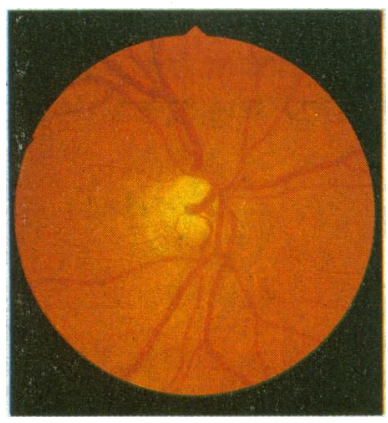

Glaucomatous cupping of optic disc.

\section{Chronic open angle glaucoma}

\section{Groups at risk:}

Older age groups

Relatives of patients

Diabetics

Extremely shortsighted patients
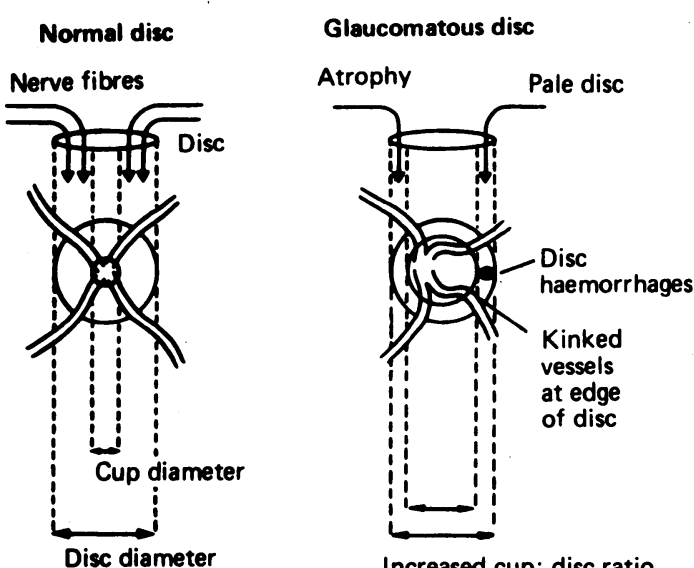

Optic disc changes in glaucoma.

\section{Acute angle closure glaucoma}

\section{Groups at risk: \\ Older patients \\ Longsighted patients}

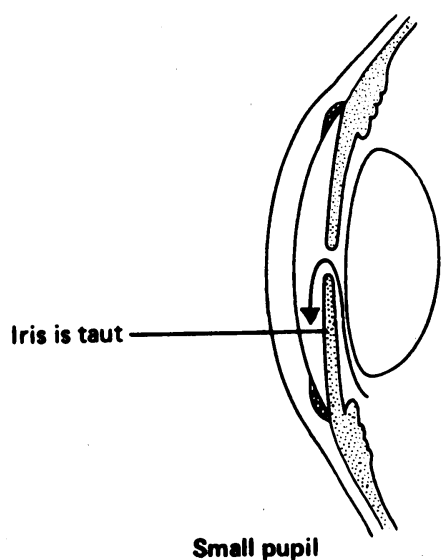

Acute angle closure glaucoma.
Venous occlusion - Raised intraocular pressure can impede blood flow in the low pressure venous system predisposing to venous occlusion.

Enlargement of the eye-In the adult no enlargement of the eye is possible because growth has ceased. In a young child, however, the eye may expand causing enlargement of the eye (buphthalmos or "ox eye"). These children may also be photophobic and have a watering eye.

Chronic open angle glaucoma is the commonest form of glaucoma and accounts for just over a 10th of all new entries on the blind registers every year. The resistance to outflow through the trabecular meshwork gradually increases for reasons that are not fully understood, and the pressure in the eye slowly increases causing damage to the nerve. There may also be other mechanisms of damage, particularly ischaemia of the optic nerve head.

Symptoms-Because the visual loss is gradual patients do not usually present until severe damage has occurred. The disease can be detected by screening high risk groups for the signs of glaucoma. At present most patients with chronic open angle glaucoma are detected by optometrists.

Groups at risk-The prevalence increases with age from $0.02 \%$ in the 40-49 age group to $10 \%$ in those aged over 80 . Relatives of patients are also at risk (one in 10), as are diabetics and extremely shortsighted people.

Signs-The eye is white and quiet. Field loss is difficult to pick up clinically until much damage has occurred. The best signs for the purpose of detection are the disc changes. Asymmetry of disc cupping is also important, as the disease is often more advanced in one eye than the other. Haemorrhages on the optic disc are a poor prognostic sign.

Acute angle closure glaucoma is probably the best known type of glaucoma as the presentation is acute and the affected eye becomes red and painful. In angle closure glaucoma apposition of the lens to the back of the iris prevents the flow of aqueous from the posterior chamber to the anterior chamber. This is more likely to occur when the pupil is semidilated at night. Aqueous then collects behind the iris and pushes it on to the trabecular meshwork preventing the drainage of aqueous from the eye, and the intraocular pressure rises rapidly. 


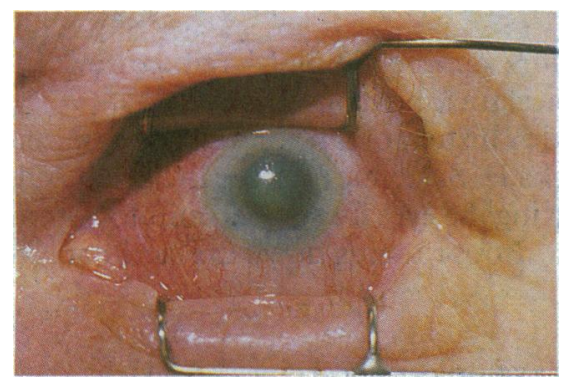

Acute angle closure glaucoma.

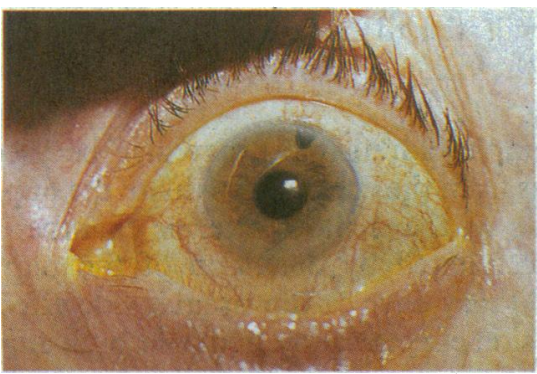

Surgical peripheral iridectomy.

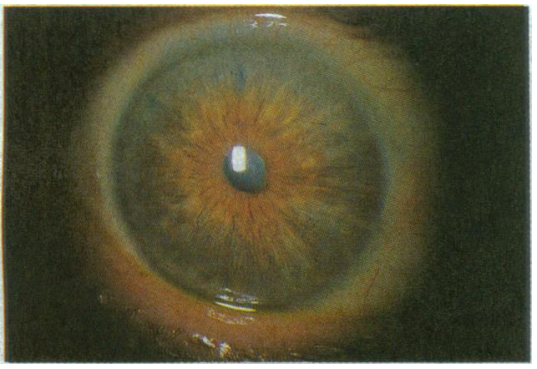

Laseriridotomies.

\section{Other types of glaucoma}

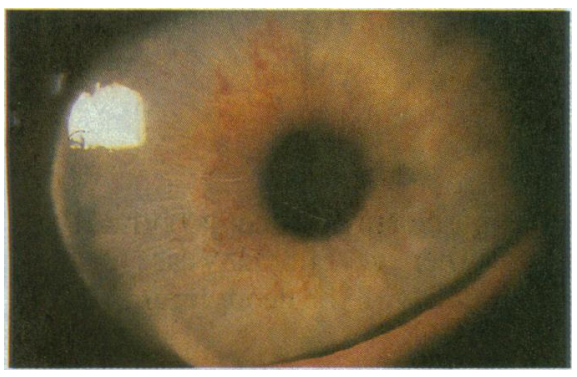

New vessels on iris causing rubeotic glaucoma.

Topical steroids may cause a change in the drainage meshwork resulting in a slow rise in intraocular pressure

- Patients may not complain of visual symptoms until severe damage has occurred
Symptoms - The eye becomes red and painful because of the rapid rise in intraocular pressure, and this is often associated with vomiting. Vision is blurred because the cornea becomes oedematous and patients may notice haloes around lights because of the dispersion of light. They may give a history of similar attacks in the past that were aborted by going to sleep. During sleep the pupil constricts and may pull the peripheral iris out of the angle.

Groups at risk - This type of glaucoma usually occurs in longsighted people, whose anterior chambers are shallow, and in the elderly, in whom the lens is larger.

Signs - The visual acuity is impaired depending on the degree of corneal oedema. The eye is red and tender to touch. The cornea is hazy because of oedema, and the pupil is semidilated and fixed to light. The attack begins with the pupil in the semidilated position and the rise in pressure makes the iris ischaemic and fixed in that position. On gentle palpation the affected eye feels much harder than the other. If the patient is seen shortly after an attack has resolved none of these signs may be present, hence the importance of the history.

Management-Emergency treatment is required if the sight of the eye is to be preserved. If it is not possible to get the patient to hospital straight away, acetazolamide (Diamox) $500 \mathrm{mg}$ should be given intravenously, and pilocarpine $4 \%$ instilled in the eye to constrict the pupil. The intraocular pressure must first be brought down medically, and then a hole must be made in the iris either surgically or with the laser in order to restore normal aqueous flow. The other eye should be similarly treated as a prophylactic measure. If the treatment is delayed adhesions may form between the iris and the cornea (peripheral anterior synechiae) and the trabecular meshwork may be damaged. A surgical drainage procedure may then be required.

If there is inflammation in the eye (anterior uveitis) adhesions may develop between the lens and the iris (posterior synechiae). These adhesions will block the flow of aqueous between the posterior and anterior chambers and result in forward ballooning of the iris and a rise in the intraocular pressure. Adhesions may also develop between the iris and the cornea (peripheral anterior synechiae) covering up the trabecular drainage meshwork. Inflammatory cells may also block up the meshwork. Topical steroids may cause a gradual asymptomatic rise in intraocular pressure which may lead to blindness.

The growth of new vessels on to the iris (rubeosis) occurs both in diabetics and after occlusion of the central retinal vein as a consequence of retinal ischaemia. These vessels also block the trabecular meshwork, causing rubeotic glaucoma, which is extremely difficult to treat.

The trabecular meshwork itself may have developed abnormally (congenital glaucoma) or been damaged by trauma to the eye. Patients who have had eye injuries have a higher chance than normal of developing glaucoma later in life. If there is a bleed in the eye after trauma the red cells may also block the trabecular meshwork. 


\section{Medical treatment}

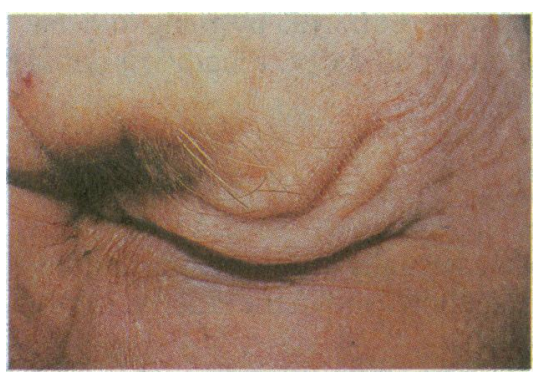

Eye closure after instilling drops to reduce systemic side effects.

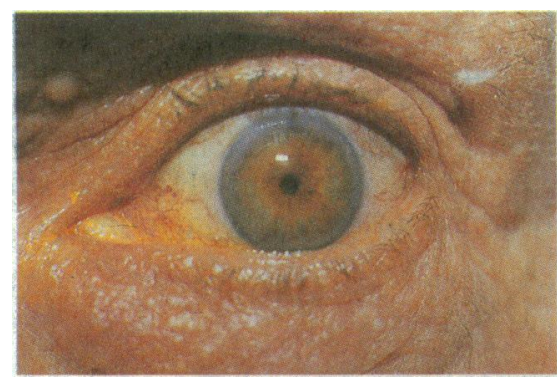

Small pupil with pilocarpine drops.
Topical treatment - $\beta$ Blockers - for example, timolol-reduce the secretion of aqueous and are the topical treatment of choice.

Contraindications to their use include a history of lung or heart disease, as the drops may cause systemic $\beta$ blockade. Systemic effects can be reduced by occlusion of the punctum or shutting the eyes for several minutes after putting in the drops, which stops the drops running down the lacrimal passages and being absorbed.

Parasympathomimetic agents-for example, pilocarpine-constrict the pupil and "pull" on the trabecular meshwork, so opening it up. The small pupil may, however, cause visual problems if central lens opacities are present. Constriction of the ciliary body causes accommodation and blurred vision in young patients. Pilocarpine should not be used if there is inflammation in the eye as the pupil will stick to the lens close to the visual axis (posterior synechiae) and affect the vision.

Sympathomimetic agents-for example, adrenaline-may also be used. They increase the outflow of aqueous from the eye but also dilate the pupil. They should not be used in patients with a shallow anterior chamber as closed angle glaucoma may be precipitated by dilatation of the pupil. Adrenaline causes local irritation and may have cardiovascular side effects due to systemic absorption. These side effects can be reduced by using a pro-drug that only converts to its active form when in the eye.

Oral or intravenous treatment -Carbonic anhydrase inhibitors-for example, acetazolamide - reduce the secretion of aqueous and are the most powerful drugs for reducing intraocular pressure. Unfortunately they have many side effects, including nausea, lassitude, and renal stones.

\section{Laser treatment}

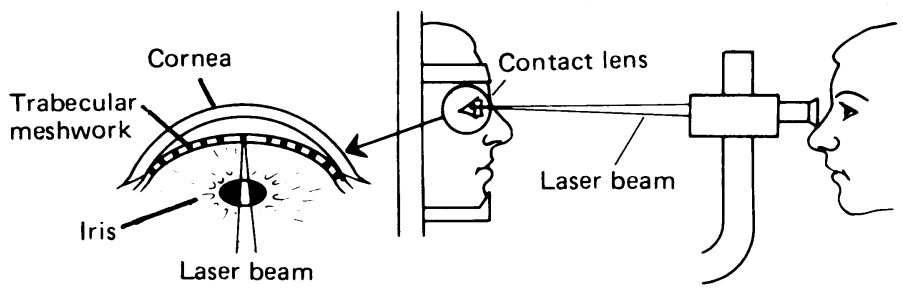

Laser trabeculoplasty.
Laser trabeculoplasty_Argon laser "burns" are applied to the trabecular meshwork. This may work by contracting one part of the meshwork, so stretching and opening up adjacent areas.

Laser iridotomy can be performed in cases of angle closure glaucoma with the neodymium YAG laser, which - unlike the argon laser-actually cuts holes in tissue rather than just burning. This procedure can be performed without opening the eye surgically.

\section{Surgical treatment}

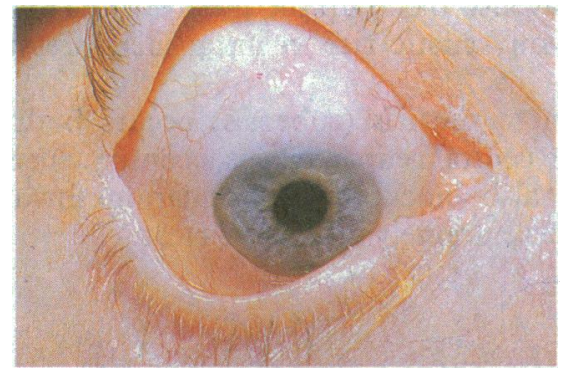

Conjunctival bleb after drainage operation.
Iridectomy is performed in cases of angle closure glaucoma both in the affected eye and prophylactically in the other eye, but many cases previously treated in this way can be treated with the neodymium YAG laser.

Drainage operation-A channel is created between the inside of the eye and the subconjunctival space, thus bypassing the blocked trabecular meshwork. A drainage "bleb" can often be seen under the upper lid. Surgery is usually performed only when medical treatment has failed to halt the progress of the disease. There is an increased incidence of cataracts after drainage operations. 